\title{
Transverse vibration of nonuniform Euler-Bernoulli beams on bounded time scales
}

\author{
Hüseyin Tuna ${ }^{\mathrm{a}^{*}}$ and Hatice Bulut ${ }^{\mathrm{a}}$ \\ a Department of Mathematics, Mehmet Akif Ersoy University, Burdur, Turkey \\ *Corresponding authorE-mail: hustuna@gmail.com
}

\section{Article Info}

Keywords: Dissipative extensions, Self adjoint extensions, A boundary value space, Boundary condition, Nonuniform beam, Time scale

2010 AMS: 74H45, 34N05, 34B20,

34B24, $47 B 25$

Received: 27 March 2018

Accepted: 24 May 2018

Available online: 30 June 2018

\begin{abstract}
In this article, we consider Euler-Bernoulli equation of transverse vibrations of nonuniform beams on bounded time scales $\mathbb{T}$. We will give a description of all maximal dissipative, maximal accretive, self adjoint and other extensions of such operators.
\end{abstract}

\section{Introduction}

The theory of symmetric extensions of a symmetric operator in a Hilbert space developed by J. von Neumann [27]. Especially, it plays a central role in spectral problems associated with formally self-adjoint linear differential operators. The problem on the description of all self adjoint extensions of a symmetric operator in terms of abstract boundary conditions was given by Calkin [25]. Later, Rofe- Beketov [28] described self adjoint extensions of a symmetric operator in terms of abstract boundary conditions with aid of linear relations. Bruk [24] and Kochubei [12] are introduced the notion of a space of boundary values. They described all maximal dissipative, accretive, self adjoint extensions of symmetric operators. This problem has been investigated by many mathematicians (see [13]-[20]). For a more comprehensive discussion of extension theory of symmetric operators, the reader is referred to [26].

The theory of time scales unifies continuous and discrete analysis. It was introduced by Hilger (see [1]). Recently, it has received a lot of attention. The study of dynamic equations on time scales has several important applications, e.g., in the study of heat transfer, insect population models, epidemic models stock market, and neural networks (see [1]-[5]).

On the other hand, transverse vibration of nonuniform beams is one of the important problems in mechanical and civil engineering. It has led to several applications in modern engineering, e.g., turbine blade, helicopter blades, satellites structure, even robotic arms etc. It has been studied by many investigators (see [29]-[43]).

In this article, we consider Euler-Bernoulli dynamic equation of transverse vibrations of nonuniform beans on bounded time scales. A space of boundary value is constructed for this operator. It is given a description all maximal dissipative, accretive, self adjoint and other extensions of such operators in terms of boundary conditions.

\section{Preliminaries}

Now, we recall some necessary fundamental concepts of time scales, and we refer to [8], [9] for more detail.

Definition 2.1. Let $\mathbb{T}$ be a time scale, i.e., a non-empty closed subset of real numbers $\mathbb{R}$. The forward jump operator $\sigma: \mathbb{T} \rightarrow \mathbb{T}$ is defined by

$$
\sigma(t)=\inf \{s \in \mathbb{T}: s>t\} \text { where } t \in \mathbb{T}
$$

and the backward jump operator $\rho: \mathbb{T} \rightarrow \mathbb{T}$ is defined by

$$
\rho(t)=\sup \{s \in \mathbb{T}: s<t\} \text { where } t \in \mathbb{T} .
$$


It is convenient to have graininess operators $\mu_{\sigma}: \mathbb{T} \rightarrow[0, \infty)$ and $\mu_{\rho}: \mathbb{T} \rightarrow(-\infty, 0]$ defined by $\mu_{\sigma}(t)=\sigma(t)-t$ and $\mu_{\rho}(t)=\rho(t)-t$, respectively. A point $t \in \mathbb{T}$ is left scattered if $\mu_{\rho}(t) \neq 0$ and left dense if $\mu_{\rho}(t)=0$. A point $t \in \mathbb{T}$ is right scattered if $\mu_{\sigma}(t) \neq 0$ and right dense if $\mu_{\sigma}(t)=0$. We introduce the sets $\mathbb{T}^{k}, \mathbb{T}_{k}, \mathbb{T}^{*}$ which are derived from the time scale $\mathbb{T}$ as follows. If $\mathbb{T}$ has a left scattered maximum $t_{1}$, then $\mathbb{T}^{k}=\mathbb{T}-\left\{t_{1}\right\}$, otherwise $\mathbb{T}^{k}=\mathbb{T}$. If $\mathbb{T}$ has a right scattered minimum $t_{2}$, then $\mathbb{T}_{k}=\mathbb{T}-\left\{t_{2}\right\}$, otherwise $\mathbb{T}_{k}=\mathbb{T}$. Finally, $\mathbb{T}^{*}=\mathbb{T}^{k} \cap \mathbb{T}_{k}$.

Definition 2.2. A function $f$ on $\mathbb{T}$ is said to be $\Delta$-differentiable at some point $t \in \mathbb{T}$ if there is a number $f^{\Delta}(t)$ such that for every $\varepsilon>0$ there is a neighborhood $U \subset \mathbb{T}$ of $t$ such that

$$
\left|f(\sigma(t))-f(s)-f^{\Delta}(t)(\sigma(t)-s)\right| \leq \varepsilon|\sigma(t)-s| \text { where } s \in U .
$$

Analogously one may define the notion of $\nabla$-differentiability of some function using the backward jump $\rho$. One can show (see [11])

$$
f^{\Delta}(t)=f^{\nabla}(\sigma(t)), \quad f^{\nabla}(t)=f^{\Delta}(\rho(t))
$$

for continuously differentiable functions.

Example 2.3. If $\mathbb{T}=\mathbb{R}$, then we have

$$
\sigma(t)=t, f^{\Delta}(t)=f^{\prime}(t)
$$

If $\mathbb{T}=\mathbb{Z}$, then we have

$$
\sigma(t)=t+1, f^{\Delta}(t)=\Delta f(t)=f(t+1)-f(t) .
$$

If $\mathbb{T}=q^{\mathbb{N}_{0}}=\left\{q^{k}: q>1, k \in \mathbb{N}_{0}\right\}$, then we have

$$
\sigma(t)=q t, f^{\Delta}(t)=\frac{f(q t)-f(t)}{q t-t} .
$$

Definition 2.4. Let $f: \mathbb{T} \rightarrow \mathbb{R}$ be a function, and $a, b \in \mathbb{T}$. If there exists a function $F: \mathbb{T} \rightarrow \mathbb{R}$ such that $F^{\Delta}(t)=f(t)$ for all $t \in \mathbb{T}^{k}$, then $F$ is a $\Delta$-antiderivative of $f$. In this case the integral is given by the formula

$$
\int_{a}^{b} f(t) \Delta t=F(b)-F(a) \text { for } a, b \in \mathbb{T} .
$$

Analogously one may define the notion of $\nabla$-antiderivative of some function.

Let $L_{\Delta}^{2}\left(\mathbb{T}^{*}\right)$ be the space of all functions defined on $\mathbb{T}^{*}$ such that

$$
\|f\|:=\left(\int_{a}^{b}|f(t)|^{2} \Delta t\right)^{1 / 2}<\infty .
$$

The space $L_{\Delta}^{2}\left(\mathbb{T}^{*}\right)$ is a Hilbert space with the inner product (see [23])

$$
(f, g):=\int_{a}^{b} f(t) \overline{g(t)} \Delta t, f, g \in L_{\Delta}^{2}\left(\mathbb{T}^{*}\right) .
$$

\section{Main results}

Let us consider Euler-Bernoulli dynamic expression of transverse vibrations of nonuniform beams

$$
l(y):=\left(E I^{*}(t) y^{\Delta \nabla}\right)^{\nabla \Delta}(t)-\rho_{0} w^{2} A^{*}(t) y(t), t \in \mathbb{T}_{1}=\mathbb{T}^{*} \cap(a, b), a<b,
$$

where $y$ is the transverse displacement, $E, \rho_{0}$ and $w$ are Young modulus, mass density, and natural frequency, respectively, $A^{*}(t)$ and $I^{*}(t)$ are the area and moment of inertia of current cross-section, respectively; $t$ is the current longitudinal coordinate of the beam, and $a$ and $b$ are the coordinates of the fixed end and the free end of the beam, respectively.

For simplicity of notation, we have

$$
\begin{aligned}
y^{[0]} & =y, \\
y^{[1]} & =y^{\Delta}, \\
y^{[2]} & =E I^{*}(t) y^{\Delta \nabla}, \\
y^{[3]} & =-\left(y^{[2]}\right)^{\nabla}, \\
y^{[4]} & =-\rho_{0} w^{2} A^{*}(t) y-\left(y^{[3]}\right)^{\Delta} .
\end{aligned}
$$

Let $y_{i}, 1 \leq i \leq 4$, be solutions of Eq. (3.1). The Wronskian of $y_{1}, y_{2}, y_{3}$ and $y_{4}$ is defined to be (see [6])

$$
W\left(y_{1}, y_{2}, y_{3}, y_{4}\right)=\left|\begin{array}{cccc}
y_{1} & y_{2} & y_{3} & y_{4} \\
y_{1}^{[1]} & y_{2}^{[1]} & y_{3}^{[1]} & y_{4}^{[1]} \\
y_{1}^{[2]} & y_{2}^{[2]} & y_{3}^{[2]} & y_{4}^{[2]} \\
y_{1}^{[3]} & y_{2}^{[3]} & y_{3}^{[3]} & y_{4}^{[3]}
\end{array}\right| .
$$


We will denote by $D o m_{\max }$ the set of all functions $y(t)$ in $L_{\Delta}^{2}\left(\mathbb{T}_{1}\right)$ such that first three $\Delta$ derivatives are locally $\Delta$ absolutely continuous in $\mathbb{T}_{1}$, and $l(y) \in L_{\Delta}^{2}\left(\mathbb{T}_{1}\right)$. We define the maximal operator $L_{\max }$ on $D o m_{\max }$ by the equality $L_{\max } y=l y$. For every $y, z \in$ Dom $_{\max }$, we have Green's formula

$$
\int_{a}^{b}(l y)(t) \overline{z(t)} \Delta t-\int_{a}^{b} y(t) \overline{(l z)(t)} \Delta t=[y, z]_{b}-[y, z]_{a}
$$

where $[y, z] t:=y^{[0]}(t) \bar{z}^{[3]}(t)-y^{[3]}(t) \bar{z}^{[0]}(t)+y^{[1]}(t) \bar{z}^{[2]}(t)-y^{[2]}(t) \bar{z}^{[1]}(t)$ (see [6] ).

Let $D o m_{\min }$ denote the linear set of all vectors $y \in D o m_{\max }$ satisfying the conditions

$$
\begin{aligned}
& y^{[0]}(a)=y^{[1]}(a)=y^{[2]}(a)=y^{[3]}(a)=0, \\
& y^{[0]}(b)=y^{[1]}(b)=y^{[2]}(b)=y^{[3]}(b)=0 .
\end{aligned}
$$

If we restrict the operator $L_{\max }$ to the set $D o m_{\min }$, then we obtain the minimal operator $L_{\min }$. It is clear that $L_{\min }^{*}=L_{\max }$, and $L_{\min }$ is a closed symmetric operator (see [6] ). Now we recall the following definitions.

Definition 3.1. A linear operator $M$ (with dense domain $D(M)$ ) acting on some Hilbert space $H$ is called dissipative (accumulative) if $\mathfrak{I}(M f, f) \geq 0(\mathfrak{I}(M f, f) \leq 0)$ for all $f \in D(M)$ and maximal dissipative (maximal accumulative) if it does not have a proper dissipative (accumulative) extension (see [14], [16]-[19]).

Definition 3.2. A triplet $\left(\mathbb{H}, \Phi_{1}, \Phi_{2}\right)$ is called a space of boundary values of a closed symmetric operator $M$ on a Hilbert space $H$ if $\Phi_{1}$ and $\Phi_{2}$ are linear maps from $D\left(M^{*}\right)$ to $H$, with equal deficiency numbers and such that:

i) For every $f, g \in D\left(M^{*}\right)$ we have

$$
\left(M^{*} f, g\right)_{H}-\left(f, M^{*} g\right)_{H}=\left(\Phi_{1} f, \Phi_{2} g\right)_{\mathbb{H}}-\left(\Phi_{2} f, \Phi_{1} g\right)_{\mathbb{H}}
$$

ii) For any $F_{1}, F_{2} \in H$ there is a vector $f \in D\left(M^{*}\right)$ such that $\Phi_{1} f=F_{1}$ and $\Phi_{2} f=F_{2}$ (see [10]). Let's define by $\Phi_{1}, \Phi_{2}$ the linear maps from $D$ to $\mathbb{C}^{4}$ by the formula

$$
\Phi_{1} y=\left(\begin{array}{c}
-y^{[0]}(a) \\
-y^{[1]}(a) \\
y^{[0]}(b) \\
y^{[1]}(b)
\end{array}\right), \Phi_{2} y=\left(\begin{array}{c}
y^{[3]}(a) \\
y^{[2]}(a) \\
y^{[3]}(b) \\
y^{[2]}(b)
\end{array}\right)
$$

Now we will state and prove a theorem.

Theorem 3.3. The triple $\left(\mathbb{C}^{4}, \Phi_{1}, \Phi_{2}\right)$ defined by (3.3) is a boundary spaces of the operator $L_{\min }$.

Proof. For every $y, z \in$ Dom $_{\max }$, we have

$$
\begin{aligned}
\left(\Phi_{1} y, \Phi_{2} z\right)_{\mathbb{C}^{4}}-\left(\Phi_{1} z, \Phi_{2} y\right)_{\mathbb{C}^{4}}= & -y^{[0]}(a) \bar{z}^{[3]}(a)-y^{[1]}(a) \bar{z}^{[2]}(a) \\
& +y^{[0]}(b) \bar{z}^{[3]}(b)+y^{[1]}(b) \bar{z}^{[2]}(b) \\
& -\left(-\bar{z}^{[0]}(a) y^{[3]}(a)-\bar{z}^{[1]}(a) y^{[2]}(a)\right) \\
& -\left(\bar{z}^{[0]}(b) y^{[3]}(b)+\bar{z}^{[1]}(b) y^{[2]}(b)\right) \\
= & y^{[0]}(b) \bar{z}^{[3]}(b)-\bar{z}^{[0]}(b) y^{[3]}(b) \\
& +y^{[1]}(b) \bar{z}^{[2]}(b)-\bar{z}^{[1]}(b) y^{[2]}(b) \\
& +\bar{z}^{[0]}(a) y^{[3]}(a)-y^{[0]}(a) \bar{z}^{[3]}(a) \\
& -y^{[1]}(a) \bar{z}^{[2]}(a)+\bar{z}^{[1]}(a) y^{[2]}(a) \\
= & {[y, z](b)-[y, z](a) . }
\end{aligned}
$$

From Green's formula (3.2), we obtain the following equation

$$
\left(\Phi_{1} y, \Phi_{2} z\right)_{\mathbb{C}^{4}}-\left(\Phi_{1} z, \Phi_{2} y\right)_{\mathbb{C}^{4}}=[y, z](b)-[y, z](a)=\left(L_{\max } y, z\right)-\left(y, L_{\max } z\right) .
$$

So, we proved the first requirement of the definition of a space of boundary values. 
Now, we will prove the second requirement of the definition of a space of boundary values. Let $u=\left(\begin{array}{l}u_{1} \\ u_{2} \\ u_{3} \\ u_{4}\end{array}\right), v=\left(\begin{array}{c}v_{1} \\ v_{2} \\ v_{3} \\ v_{4}\end{array}\right) \in \mathbb{C}^{4}$. Then the vector-valued function

$$
y(t)=\alpha_{1}(t) u_{1}+\alpha_{2}(t) v_{1}+\alpha_{3}(t) u_{2}+\alpha_{4}(t) v_{2}+\alpha_{5}(t) u_{3}+\alpha_{6}(t) v_{3}+\alpha_{7}(t) u_{4}+\alpha_{8}(t) v_{4},
$$

where $\alpha_{i}(t) \in H(i=1, \ldots, 8)$ satisfy the conditions

$$
\begin{array}{lccc}
\alpha_{1}^{[0]}(a)=1 & \alpha_{1}^{[1]}(a)=0 & \alpha_{1}^{[2]}(a)=0 & \alpha_{1}^{[3]}(a)=0 \\
\alpha_{2}^{[0]}(a)=0 & \alpha_{2}^{[1]}(a)=0 & \alpha_{2}^{[2]}(a)=0 & \alpha_{2}^{[3]}(a)=1 \\
\alpha_{3}^{[0]}(a)=0 & \alpha_{3}^{[1]}(a)=-1 & \alpha_{3}^{[2]}(a)=0 & \alpha_{3}^{[3]}(a)=0 \\
\alpha_{4}^{[0]}(a)=0 & \alpha_{4}^{[1]}(a)=0 & \alpha_{4}^{[2]}(a)=1 & \alpha_{4}^{[3]}(a)=0 \\
\alpha_{5}^{[0]}(a)=0 & \alpha_{5}^{[1]}(a)=0 & \alpha_{5}^{[2]}(a)=0 & \alpha_{5}^{[3]}(a)=0 \\
\alpha_{6}^{[0]}(a)=0 & \alpha_{6}^{[1]}(a)=0 & \alpha_{6}^{[2]}(a)=0 & \alpha_{6}^{[3]}(a)=0 \\
\alpha_{7}^{[0]}(a)=0 & \alpha_{7}^{[1]}(a)=0 & \alpha_{7}^{[2]}(a)=0 & \alpha_{7}^{[3]}(a)=0 \\
\alpha_{8}^{[0]}(a)=0 & \alpha_{8}^{[1]}(a)=0 & \alpha_{8}^{[2]}(a)=0 & \alpha_{8}^{[3]}(a)=0
\end{array}
$$

and

belongs to the set $D m_{\max }$ and $\Phi_{1} y=u, \Phi_{2} y=v$

$$
\begin{array}{cccc}
\alpha_{1}^{[0]}(b)=0 & \alpha_{1}^{[1]}(b)=0 & \alpha_{1}^{[2]}(b)=0 & \alpha_{1}^{[3]}(b)=0 \\
\alpha_{2}^{[0]}(b)=0 & \alpha_{2}^{[1]}(b)=0 & \alpha_{2}^{[2]}(b)=0 & \alpha_{2}^{[3]}(b)=0 \\
\alpha_{3}^{[0]}(b)=0 & \alpha_{3}^{[1]}(b)=0 & \alpha_{3}^{[2]}(b)=0 & \alpha_{3}^{[3]}(b)=0 \\
\alpha_{4}^{[0]}(b)=0 & \alpha_{4}^{[1]}(b)=0 & \alpha_{4}^{[2]}(b)=0 & \alpha_{4}^{[3]}(b)=0 \\
\alpha_{5}^{[0]}(b)=-1 & \alpha_{5}^{[1]}(b)=0 & \alpha_{5}^{[2]}(b)=0 & \alpha_{5}^{[3]}(b)=0 \\
\alpha_{6}^{[0]}(b)=0 & \alpha_{6}^{[1]}(b)=0 & \alpha_{6}^{[2]}(b)=0 & \alpha_{6}^{[3]}(b)=1 \\
\alpha_{7}^{[0]}(b)=0 & \alpha_{7}^{[1]}(b)=1 & \alpha_{7}^{[2]}(b)=0 & \alpha_{7}^{[3]}(b)=0 \\
\alpha_{8}^{[0]}(b)=0 & \alpha_{8}^{[1]}(b)=0 & \alpha_{8}^{[2]}(b)=1 & \alpha_{8}^{[3]}(b)=0
\end{array}
$$

Corollary 3.4. For any contraction $T$ in $\mathbb{C}^{4}$ the restriction of the operator $L_{\max }$ to the set of functions $y \in D_{\max }$ satisfying either

$$
(T-I) \Phi_{1} y+i(T+I) \Phi_{2} y=0
$$

or

$$
(T-I) \Phi_{1} y-i(T+I) \Phi_{2} y=0
$$

is respectively the maximal dissipative and accretive extension of the operator $L_{\min }$. Conversely, every maximal dissipative (accretive) extension of the operator $L_{\min }$ is the restriction of $L_{\max }$ to the set of functions $y \in \operatorname{Dom}_{\max }$ satisfying (3.4) ( (3.5)), and the extension uniquely determines the contraction $T$. If $T$ is an isometry in $\mathbb{C}^{4}$, then the conditions (3.4) ( (3.5)) describe the maximal symmetric extensions of $L_{\text {min }}$ in $L_{\Delta}^{2}\left(\mathbb{T}_{1}\right)$.

The general form of dissipative and accretive extensions of an operator $L$ is given by the conditions

$$
\begin{aligned}
& T\left(\Phi_{1} y+i \Phi_{2} y\right)=\Phi_{1} y-i \Phi_{2} y, \Phi_{1} y+i \Phi_{2} y \in \operatorname{Dom}(T), \\
& T\left(\Phi_{1} y-i \Phi_{2} y\right)=\Phi_{1} y+i \Phi_{2} y, \Phi_{1} y-i \Phi_{2} y \in \operatorname{Dom}(T),
\end{aligned}
$$

respectively, where $T$ is a linear operator with

$$
\|T f\| \leq\|f\|, f \in \operatorname{Dom}(T)
$$

\section{Conclusion}

In this paper, we have considered Euler-Bernoulli equation of transverse vibrations of nonuniform beams on bounded time scales $\mathbb{T}$.. In this context, we have constructed a space of boundary values of the minimal operator and described all maximal dissipative, maximal accretive, self-adjoint extensions of of such operators.

\section{References}

[1] Hilger S., Analysis on measure chains-a unified approach to continuous and discrete calculus, Results in Math., (1990), 1818 - 1856.

[2] Agarwal R. P., Bohner M., and Li W.-T., Nonoscillation and Oscillation Theory for Functional Differential Equations, vol. 267 of Monographs and Textbooks in Pure and Applied Mathematics, Marcel Dekker, New York, NY, USA, 2004.

[3] Jones M. A., Song B. and Thomas D. M., "Controlling wound healing through debridement," Mathematical and Computer Modelling, vol. 40, no. 9-10, pp. 1057-1064, 2004.

[4] Spedding V., "Taming nature’s numbers," New Scientist, vol. 179, no. 2404, pp. 28-31, 2003.

[5] Thomas D. M., Vandemuelebroeke L. and Yamaguchi K., "A mathematical evolution model for phytoremediation of metals," Discrete and Continuous Dynamical Systems. Series B, vol. 5, no. 2, pp. 411-422, 2005.ker, Florida, 2004. 
[6] Anderson D. R. , Guseinov Gusein Sh., and Hoffacker J. , Higher-order self-adjoint boundary-value problems on time scales, J. Comput. Appl. Math., $194,2,(2006) 309-342$.

[7] Atici Merdivenci F. and Guseinov Gusein Sh., On Green's functions and positive solutions for boundary value problems on time scales, J. Comput. Appl. Math., 141,1 - 2,(2002) $75-99$.

[8] Bohner M. and Peterson A., Dynamic Equations on Time Scales, Birkhäuser, Boston, 2001.

[9] Bohner M. and Peterson A., (Eds.), Advances in Dynamic Equations on Time Scales, Birkhäuser, Boston, 2003.

[10] Gorbachuk M. L. and Gorbachuk V.I. , Boundary Value Problems for Operator Differential Equations, Naukova Dumka, Kiev, 1984 ; English transl. 1991,Birkhauser Verlag.

[11] Guseinov Gusein Sh., Self-adjoint boundary value problems on time scales and symmetric Green's functions, Turkish J. Math., 29 (4), (2005) 365 - 380.

[12] Kochubei A. N., Extensions of symmetric operators and symmetric binary relations, Mat. Zametki 17,(1975) 41 - 48; English transl. in Math. Notes $17,(1975) 25-28$.

[13] M.G. Krein, On the indeterminate case of the Sturm-Liouville boundaryvalue problem in the interval (0, $\infty)$ ', Akad. Nauk SSSR Ser. Mat. $16,(1952), 292-324$

[14] F.G. Maksudov and B.P. Allahverdiev, On the extensions of Schrödinger operators with a matrix potentials, Dokl. Akad. Nauk 332, no.1, (1993), 18 20;English transl. Russian Acad. Sci. Dokl. Math. 48 (1994), no.2, 240 - 243.

[15] M. M. Malamud and V. I. Mogilevskiy, On extensions of dual pairs of operators, Dopov. Nats Akad. Nauk. Ukr. (1997),no. 1,30 - 37.

[16] B.P. Allahverdiev, Extensions of symmetric singular second-order dynamic operators on time scales. Filomat 30 (2016), no. 6, 1475-1484.

[17] B.P. Allahverdiev, Extensions of symmetric infinite Jacobi operator. Linear Multilinear Algebra 62 (2014), no. 9, 1146-1152.

[18] B.P. Allahverdiev, Extensions of symmetric second-order difference operators with matrix coefficients. J. Difference Equ. Appl. 19 (2013), no. 5, 839-849.

[19] A. Canoğ lu and B.P. Allahverdiev, Selfadjoint and dissipative extensions of a symmetric Schrödinger operator. Math. Balkanica (N.S.) 17 (2003), no. $1-2,113-120$.

[20] H. Tuna and B. P. Allahverdiev, Dissipative Extensions of Fourth Order Differential Operators, Thai Journal of Mathematics, Vol. 16 (1), (2018), $275-285$.

[21] Lakshmikantham V., Sivasundaram S. and Kaymakcalan B., Dynamic Systems on Measure Chains, Kluwer Academic Publishers, Dordrecht, 1996.

[22] Naimark M. A., Linear Differential Operators, 2nd edn., 1968, Nauka, Moscow, English transl. of 1st. edn., 1,2, 1969, New York.

[23] Rynne B. P., $L^{2}$ spaces and boundary value problems on time-scales, J. Math. Anal. Appl. 328, (2007) 1217 - 1236.

[24] V.M. Bruk, On a class of boundary -value problemswith a spectral parameter in the boundary conditions, Mat. Sb.,100,(1976),210 - 216.

[25] J. W. Calkin, Abstract boundary conditions, Trans. Amer. Math. Soc.,Vol 45,No. 3, (1939), $369-442$.

[26] M.L. Gorbachuk, V.I. Gorbachuk and A.N. Kochubei, 1989. The theory of extensions of symmetric operators and boundary-value problems for differential equations', Ukrain. Mat. Zh. 41,(1989), 1299-1312; English transl. in Ukrainian Math. J. 41(1989), 1117 - 1129.

[27] J. von Neumann, Allgemeine Eigenwertheorie Hermitischer Functionaloperatoren, Math. Ann. 102, (1929), 49 - 131.

[28] F.S. Rofe-Beketov, Self-adjoint extensions of differential operators in a space of vector valued functions', Dokl. Akad. Nauk SSSR 184, (1969), 1034 1037;English transl. in Soviet Math. Dokl. 10(1969), $188-192$

[29] H.D. Conway, J.F. Dubil, Vibration frequencies of truncated wedge and cone beam, Journal of Applied Mechanics 32E (1965) 932-935.

[30] J.J. Mabie, C.B. Rogers, Traverse vibrations of tapered cantilever beams with end support, Journal of Acoustical Society of America 44 (1968) 1739-1741.

[31] M.A. De Rosa, N.M. Auciello, Free vibrations of tapered beams with flexible ends, Computers \& Structures 60 (2) (1996) $197-202$.

[32] H.D. Conway, E.C.H. Becker, J.F. Dubil, Vibration frequencies of tapered bars and circular plates, Journal of Applied Mechanics June (1964) 329-331.

[33] E.T. Cranch, A. Adler, Bending vibrations of variable section beams, Journal of Applied Mechanics March (1956) $103-108$.

[34] N.M. Auciello, G. Nole, Vibrations of a cantilever tapered beam with varying section properties and carrying a mass at the free end, Journal of Sound and Vibration, 214 (1) (1998) 105-119.

[35] H.C. Wang, Generalized hypergeometric function solutions on the transverse vibrations of a class of non-uniform beams, Journal of Applied Mechanics 34E (1967) 702-708.

[36] D. Storti, Y. Aboelnaga, Bending vibrations of a class of rotating beams with hypergeometric solutions, Journal of Applied Mechanics, 54 (1987)

[37] D.I. Caruntu, On nonlinear vibration of nonuniform beam with rectangular cross-section and parabolic thickness variation, Solid Mechanics and its Applications, Vol. 73, Kluwer Academic Publishers, Dordrecht, Boston, London, 2000, pp. 109-118.

[38] D.I. Caruntu, Relied studies on factorization of the differential operator in the case of bending vibration of a class of beams with variable crosssection,Revue Roumaine des Sciences Techniques, Série de Mecanique Appliquee, 41 (5-6) (1996) 389-397.

[39] D.I. Caruntu, Dynamic modal characteristics of transverse vibrations of cantilevers of parabolic thickness. Mechanics Research Communications 36 : (2009)391-404

[40] S. Naguleswaran, The vibration of a "complete" Euler-Bernoulli beam of constant depth and breadth proportional to axial co-ordinate raised to a positive exponents, Journal of Sound and Vibration, 187 (2) (1995) 311-327.

[41] S. Naguleswaran, A direct solution for the transverse vibration of Euler-Bernoulli wedge and cone beams, Journal of Sound and Vibration, 172 (3) (1994) 289-304.

[42] A.D. Wright, C.E. Smith, R.W. Thresher, J.L.C. Wang, Vibration modes of centrifugally stiffened beam, Journal of Applied Mechanics, 49 (1982)

[43] Q. Wang, Sturm-Liouville equation for free vibration of a tube-in-tube tall building, Journal of Sound and Vibration, 191 (3) (1996), 349-355. 